\title{
How quickly does phonological-syntactic information decay?
}

\author{
HIRAM H. BROWNELL, ALFONSO CARAMAZZA, and MARK H. BRADSHAW \\ The Johns Hopkins University, Baltimore, Maryland 21218
}

\begin{abstract}
An experiment is reported that investigates the kinds of information people remember about sentences. During an acquisition phase, subjects processed sentences semantically in order to perform a rating task. Later, when tested for recognition, subjects false alarmed to novel sentences that shared semantic content with the acquisition sentences. They retained, however, significant amounts of detail about the surface form in which the semantic content was first encountered. The discrepancies between this experiment and earlier work is discussed in terms of different memory stores for different kinds of sentence information.
\end{abstract}

In recent years, several researchers have drawn our attention to the question of what people remember about sentences (Anderson \& Bower, 1974; Bransford \& Franks, 1971; Foss \& Harwood, 1975; Franks \& Bransford, 1972; Green, 1975). In this research, one central goal has been to determine an appropriate unit of analysis. Of special interest in this regard is the work of Bransford and Franks (1971). Rather than look at memory for single words or simple sentences, these researchers developed an experimental paradigm to investigate memory for "holistic, semantic ideas." Their paradigm consisted of an acquisition phase and a test phase. During the acquisition phase, subjects were presented with a randomized sequence of sentences and were required to process the sentences sufficiently to answer elliptical questions about them. A crucial feature of these sentences was that the semantic content of the individual sentences, when integrated without respect to the serial order in which they appeared, formed cohesive ideas or thoughts. For example, the following sentences might have appeared at various positions in the acquisition list: (1) The rock rolled down the mountain. (2) The rock crushed the hut. (3) The tiny hut was at the edge of the woods. (4) The hut was tiny. (5) The hut was at the edge of the woods. When integrated, these individual sentences express the following "holistic semantic idea": (6) The rock which rolled down the mountain crushed the tiny hut at the edge of the woods. The holistic idea did not appear in the list of acquisition sentences. Subjects were then presented with a second list of sentences, some of which had appeared in the first (acquisition) list and some of which had not. The subjects' task was to respond OLD if a sentence had appeared on the earlier list, NEW if it had not, and to give a confidence rating. An important variable in

We wish to thank Barry Sagalow for preparing the stimuli and for assisting in the data analysis. Reprint requests should be addressed to Alfonso Caramazza, Department of Psychology, The Johns Hopkins University, Charles and 34th Streets, Baltimore, Maryland 21218. the experiment was that some NEW sentences were semantically related to the holistic ideas expressed by sentences in the acquisition list. Using the examples given earlier (Sentences 1-5) as members of the acquisition list, one might have encountered the following (NEW) sentence on the second (test) list: (7) The rock which rolled down the mountain crushed the hut. Although the exact form of Sentence 7 never appeared on the first list, the semantic content of the sentence is OLD. Two major results of the Bransford and Franks experiments were that in the test phase subjects (1) false alarmed more to NEW sentences as the semantic content approached that of the holistic idea, and (2) were unable to discriminate OLD from NEW sentences when they expressed as much (similar amounts) of a holistic idea.

To deal with these results, Bransford and Franks suggested that the most appropriate unit of analysis was predominantly semantic. We felt, however, that their results left a critical question unanswered: Why is it that in some conditions subjects have shown relatively better memory for semantic information, and in other conditions better memory for nonsemantic information?

Recently, Green (1975) showed that differences in experimental tasks could affect the nature of subjects' representations of stimulus sentences. One group of subjects (in Experiment 1) memorized sentences and a second group produced semantically consistent continuations of the same sentences. After presentation of each sentence and before responding according to the respective experimental tasks (i.e., recalling the sentence verbatim, producing a continuation), subjects decided whether or not a probe word had appeared in the sentence. One result was that negative probes (i.e., probes that did not appear in the sentences) were easier or harder to reject as a function of experimental task. Those subjects who simply memorized sentences found it easier to reject (implying greater psychological difference, e.g., see Rips, Shoben, \& Smith, 1973) semantically related probes than to reject phonologically related probes. On the other hand, those subjects who produced 
continuations found it harder to reject semantically related probes than phonologically related probes. To account for these results, Green (1975) suggested that memorizing a sentence biased subjects to retain the meanings of its individual words separately, while producing a continuation (a task which requires a deeper level of analysis) biased them to combine the meanings of its words into a single unit. Two different kinds of information were apparently stored, depending on the experimental task. Other researchers as well (Yates \& Caramazza, Note 1) have discussed the need for two separate types of sentence memory, (1) an abstract semantic interpretation and (2) a more veridical phonological-syntactic representation. These two types of memory representations may have different decay rates and, in general, a fair degree of independence, and they may be differentially sensitive to different sources of interference.

The design of the present experiment incorporates the assumption that subjects have different (and probably separate, although this does not concern us here) memories for the different kinds of information in a sentence, and that the experimental task requirements can affect these memories differentially. Accordingly, the appropriate unit of analysis to describe sentential memory must be determined by both of the above considerations. Recall that in the Bransford and Franks paradigm, subjects were not aware that they would be tested for sentence memory. Also, after each sentence presentation in the acquisition phase, subjects answered an elliptical question about the sentence. In these two important details, the Bransford and Franks (1971) and Green (1975) paradigms (the continuation group only) were quite similar and, we feel, analogous. In the present experiment we departed from the Bransford and Franks paradigm in only a few respects and obtained strikingly different results. First, subjects were told at the start of the session that they would be tested for sentence memory. Second, semantically unrelated "filler" sentences were inserted after each stimulus sentence in the acquisition list, and the color-naming task used by Bransford and Franks was omitted. (It was felt that this last change would not significantly alter the experimental task.) Also, subjects were required to rate the meaningfulness of each sentence in the acquisition list, but they were not required to answer elliptical questions (or produce continuations). This change was made because subjects in Bransford and Franks' and Green's experiments (Green's continuation group only) must have recoded the semantic content of each acquisition sentence to perform the experimental task. Such recoding could quite plausibly have generated interference specific to memory for phonological-syntactic information or for surface form in general. In other respects, the semantic rating task was thought to be analogous to answering elliptical questions insofar as it forced subjects to process the semantic content of each sentence (Foss
\& Harwood, 1975). Conceptually, this new paradigm is a hybrid. Subjects were aware that they had to remember the sentences (although they were not simply memorizing the wording of the sentences, as will be made clear from the results of the experiment); they also had to process the sentences' meaning; most important, there was no obvious task-produced interference which would differentially affect memory for either semantic or surface information.

\section{METHOD}

\section{Subjects}

Forty-eight Johns Hopkins University undergraduates enrolled in an introductory level psychology course participated as part of a class requirement.

\section{Stimuli}

Stimuli were constructed from four "ideas," each of which was first broken down into four simple, declarative sentences which together exhausted the semantic content of the original "idea." The simple, declarative sentences were then recombined in a variety of ways: Stimulus sentences were constructed from one, two, three, or four of the simple sentences from a single idea. (From this point on, stimulus sentences constructed from one, two, three, or four simple sentences will be referred to as ones, twos, threes, and fours, respectively.) In this way, 12 sentences ( 1 four, 3 threes, 4 twos, and 4 ones) were constructed from each of the four ideas, making a total of 48 stimulus sentences. An example of an idea and the possible combinations of the four component sentences are shown in Table 1.

Acquisition sentences. Six sentences (two threes, two twos, two ones) were selected from each idea, such that they (the set of six) exhausted the semantic content of the idea. In addition, 24 "filler" sentences were constructed which were semantically unrelated to the stimulus sentences and which varied in meaningfulness. Altogether, 48 sentences ( 24 stimulus and 24 filler) were presented in the acquisition phase.

Test sentences. Twenty-ight sentences were presented in the test phase. The remaining 24 stimulus sentences ( 1 four, 1 three, 2 twos, 2 ones from each idea) were NEW sentences, and 4 acquisition sentences ( 1 three, 1 two, 2 ones) were OLD.

Procedure

Acquisition phase. Subjects were told that they were going

Table 1 Stimulus Sentences Constructed from Idea 1

Idea The man by the window waved to the smiling people on the street.

Four The man by the window waved to the smiling people on the street.

Three The man by the window waved to the smiling people. The man by the window waved to the people on the street. The man waved to the smiling people on the street.

Two The man by the window waved to the people.

The man waved to the smiling people.

The smiling people were on the street.

The man waved to the people on the street.

One The man was by the window.

The man waved to the people.

The people were smiling.

The people were on the street. 
to be tested for memory of the sentences they were about to hear. The 48 acquisition sentences were read aloud (randomly mixed with filler sentences) to the subjects. At the end of each sentence there was a brief pause, during which subjects rated its meaningfulness. Upon completion of the list, subjects were asked to turn over their answer sheets and number them from 1 to 28 .

Test phase. After subjects completed the numbering task (approximately $5 \mathrm{~min}$ ), the 28 test sentences were read aloud. After each sentence there was a brief pause, during which subjects indicated whether the sentence was OLD (by answering "yes" they had seen it in the acquisition list) or NEW (by answering "no" they had not seen it before) and how confident ( 1 , not at all confident, to 7 , very confident) they were of their response.

\section{RESULTS AND DISCUSSION}

Prior to any analysis, confidence ratings associated with "no" responses were assigned negative values (e.g., a "no" response with a confidence rating of 5 was coded as -5). Each subject's mean response to each sentence type was then computed based on confidence ratings. These means broken down by sentence type and OLD and NEW are shown in Table 2, Differences between means were tested by sign tests.

As in the Bransford and Franks experiments, subjects frequently false alarmed to NEW sentences; the mean response was greater than zero for 39 out of 48 subjects, 43 out of 48,45 out of 48 , and 26 out of 47 for fours, threes, twos, and ones, respectively. Except for the ones $(p>.10)$, these orderings are all different from chance $(\mathrm{p}<.001)$. This general pattern replicates earlier work (i.e., Bransford \& Franks, 1971) and, presumably, indicates the same integrative memory for semantic information. The detailed pattern for the NEW sentences is less clear due to a lack of sensitivity in the design, and cannot be readily interpreted. The only reliable difference is that 40 out of 47 subjects were more confident they had seen NEW twos previously than NEW ones $(p<.001)$.

The most striking result in the data is that subjects consistently responded "yes" to OLD sentences with more confidence that to NEW sentences; 40 out of 47 , 35 out of 48 , and 38 out of 48 subjects showed this ordering for ones, twos, and threes, respectively $(\mathrm{p}<$ .005 for all comparisons). This result clearly indicates that subjects did retain considerable information about the surface form of the acquisition sentences. Recall that they were required to process the sentences semantically, but they were not required to perform any task that would produce interference for phonological-syntactic information.

The results reported here demonstrate the existence
Table 2

Mean Confidence Ratings for Test Sentences

\begin{tabular}{lcccr}
\hline $\begin{array}{l}\text { Sentence } \\
\text { Type }\end{array}$ & Four & Three & Two & One \\
\hline OLD & & 5.31 & 2.83 & 2.36 \\
NEW & 2.46 & 2.95 & 2.37 & .02 \\
\hline
\end{tabular}

Note-Positive values indicate a "yes" response.

of a fairly durable memory for the surface form of sentences. Also, our results, as well as those reported by other researchers, demonstrate that there is memory for specifically semantic information. The issue which we have tried to address is simply that there are (at least) two kinds of information stored in memory: an abstract semantic representation and a more veridical phonologicalsyntactic representation. What is needed for perfect recognition or recall is an appropriate confluence of these two representations. Since the more fragile veridical representation decays more rapidly and is easily subject to interference, it is often claimed that this information is lost almost immediately after input. However, as our results demonstrate, this type of information can, under appropriate conditions, be available for as much as 10 or $15 \mathrm{~min}$. Finally, the relative strength of these different memories can be successfully interpreted within a traditional theory of interference. Specifically, interference generated by specific experimental tasks can greatly affect what information is retained.

\section{REFERENCE NOTE}

1. Yates. J. B.. \& Caramazza. A. Prompted recall of grammatical cases. Paper presented at the meeting of the Eastern Psychological Association. Philadelphia. April 1974.

\section{REFERENCES}

ANderson. J. R., \& Bower, G. H. Human associative memory. Washington, D.C: Hemisphere, 1974.

Bransford. J. D., \& Franks, J. J. The abstraction of linguistic ideas. Cognitive Psychology, 1971, 2, 331-350.

Foss, D. J., \& HaRwood, D. A. Memory for sentences: Implications for human associative memory. Journal of Verbal Learning and Verbal Behavior, 1975, 14, 1-16.

Franks. J. J.. \& BRANSFORD. J. D. The acquisition of abstract ideas. Journal of Verbal Learning and Verbal Behavior, 1972. 11. $311-315$.

GREEN. D. W. The effects of task on the representation of sentences. Journal of Verbal Learning and Verbal Behavior, 1975. 14. $275-283$.

Rips. L. J., Shoben. E. J.. \& Smith, E. E. Semantic distance and the verification of semantic relations. Journal of Verbal Learning and Verbal Behavior, 1973, 12, 1-20.

(Received for publication August 24. 1977.) 